www.jmscr.igmpublication.org

Index Copernicus Value: 79.54

ISSN (e)-2347-176x ISSN (p) 2455-0450

crossref DOI: https://dx.doi.org/10.18535/jmscr/v7i4.164

\title{
Evaluating the Role of Statins in Patients with non-Alcoholic Fatty Liver Disease (NAFLD)
}

\author{
Authors \\ Dr Gagan Gunjan ${ }^{1}$, Dr P.K.Agrawal ${ }^{2}$, Dr Krishna Baruah ${ }^{3}$ \\ ${ }^{1}$ MD Gen. Medicine \\ ${ }^{2}$ Prof. \& HOD, Medicine, KMCH \\ ${ }^{3}$ Prof. \& Medicine $\mathrm{KMCH}$
}

\section{Introduction}

Non-alcoholic fatty liver disease is an emerging liver disease in Indian and Western countries. It is defined as fatty infiltration of liver with a spectrum of disorders ranging from simple fatty liver to non alcoholic staeatohepatitis to fibrosis/cirrhosis attributable to various risk factors like Obesity, hyperlipidaemia, Type2 DM and insulin resistance. ${ }^{1,2}$ Fatty liver is not an unusual finding nowadays-being recognized in 30$40 \%$ of general population, $10-20 \%$ of normal weight individuals and $70-80 \%$ of obese individuals. $^{4,5}$

It is one of the most frequent causes of incidental elevation of serum liver enzymes. Dyslipidaemia is frequently observed in patients with nonalcoholic fatty liver disease and treatment with statins play a critical role in overall management of these patients. NAFLD is now recognized as the most prevalent chronic liver disease, a situation that is not going to change soon and statins play a crucial role in lowering the risk of cardiovascular events shown in several clinical trials. Global prevalence of NAFLD is estimated to be between $25 \%$ and $33 \%$. ${ }^{9,10}$

\section{Objective}

The primary objective is to demonstrate beneficial role of statins in patients of NAFLD with minimal effect on liver transaminases (ALT/AST) thus making it a promising drug for the same.

\section{Material and Method}

This study was carried out at Katihar Medical College. During the said period a total of 100 patients were selected for study based on USG finding of FATTY LIVER GRADE-1,2,3, along with raised liver enzymes (AST/ALT) after ruling out other causes like: alcoholic misuse, chronic hepatitis B, hepatitis c, etc. Treatment was initiated with structured care with statin versus usual care. In the structured group statin was initiated at low dose and gradually increased to higher dose. Usual care included lifestyle changes, low fat diet, weight loss, exercise. All patients were evaluated with repeat USG and LIVER function tests at 3 months and 6 months.

USG grading of NAFLD ${ }^{3,34-38}$

Grade I (mild): Mild increase in the fine echoes in the hepatic parenchyma with normal visualisation of the diaphragm and intrahepatic vessel borders. 
Grade II (Moderate): Moderate diffuse increase in fine echoes with mild impaired visualisation of the intrahepatic vessels and diaphragm. Grade III (severe): Marked increase in fine echoes with poor or non visualisation of the intrahepatic vessel borders, diaphragm and posterior portion of the right lobe the liver.

\section{Review of Literature}

As defined by the American Association for the Study of Liver Diseases, American College of Gastroenterology, and the American Gastroenterological Association, nonalcoholic fatty liver disease (NAFLD) is the presence of $\geqslant 5 \%$ hepatic steatosis in the absence of secondary causes of fat accumulation, including heavy alcohol consumption, treatment with steatogenic medications, and genetic causes of hepatic fat deposition. ${ }^{1}$ Nonalcoholic fatty liver disease is further histologically divided into nonalcoholic steatohepatitis (NASH) associated with inflammation, ballooning of hepatocytes, and fibrosis, or nonalcoholic fatty liver (NAFL), which is not associated with hepatocellular damage. ${ }^{1,11}$ Serum aminotransferases may be elevated or within the upper limit of normal (ULN). ${ }^{1}$ The clinical implications of NAFLD include progression to cirrhosis, hepatocellular carcinoma (HCC), liver failure requiring liver transplantation, and increased risk of cardiovascular disease (CVD). ${ }^{1}$

Hyperlipidaemia is frequently associated with NAFLD. Most patients with moderately elevated ALT levels have 'atherogenic dyslipidaemia', which is characterized by increased serum triglycerides, low HDL cholesterol and the presence of small, dense LDL particles, a common finding also in insulin resistance and metabolic syndrome. "Atherogenic dyslipidaemia" is frequently associated with other features of metabolic syndrome such as obesity, diabetes mellitus, and hypertension. Statins have antiinflammatory, anti-oxidant and anti-thrombotic effects that are independent of their lipid-lowering activity. ${ }^{12,13}$ Therefore, they have been proposed for the treatment of NAFLD and NASH, since in these conditions both inflammation and oxidative stress play an important pathogenetic role. ${ }^{14}$

Aggressive treatment of dyslipidaemia plays a critical role in the overall management of patients with NAFLD. Statins are the mainstay of lipidlowering drug therapy in patients with hyperlipidaemia. All statins appear to be effective in lowering cholesterol levels in patients with NAFLD. ${ }^{15}$ However, there is concern that patients with NAFLD or NASH and hyperlipidaemia who are treated with statins could develop serum ALT elevation or a further increase of already elevated enzymes. Therefore, in clinical practice, management of dyslipidaemia in patients with NAFLD has been often a matter of concern and under-treatment with statin therapy because of potential liver damage.

During statin treatment, an asymptomatic elevation in ALT should not be considered a sign of ongoing liver disease or injury. The term of "transaminitis" has been proposed to describe the situation of liver enzyme leakage without hepatotoxic consequences. ${ }^{16}$ Thus, "transaminitis" may explain many of the serum ALT elevations seen in patients treated with statins. There is general agreement that ALT is more useful than aspartate aminotransferase (AST) to reveal possible hepatotoxicity. Moreover, ALT elevations should be confirmed in subsequent determinations, as a single ALT elevation is more suggestive for "transaminitis" than for liver damage. $^{16}$

Various studies like WOSCOPS (West of Scotland Coronary Prevention Study) ${ }^{17}$, the Cholesterol And Recurrent Events (CARE) study ${ }^{18}$, and the Long-term Intervention with Pravastatin in Ischemic Disease (LIPID) study ${ }^{19}$. have been carried out to evaluate potential safety issues. These studies indicated that statins at lowto-moderate doses, are not associated with a significant risk of liver function test (LFT) abnormalities and that LFT monitoring, other than prior to starting therapy, is not warranted in patients taking a low-to-moderate dose. ${ }^{20}$ 


\section{Observation \& Discussion}

In our study there was a considerable difference between the structured group receiving statins and the usual group not receiving statin. The relationship between statin use, hepatic triglycerides and serum ALT levels were also examined. We observed that use of statins were not associated with high frequency of serum ALT abnormalities, even in those with hepatic steatosis. Similar findings have been reported by Alsheikh Ali etal ${ }^{21}$ and Dale KM et al. ${ }^{22}$ Overwhelming data now exist that statins are safe in the primary and secondary prevention of cardiovascular events.

A 2011 comprehensive review of the safety and efficacy of statins in patients with chronic liver disease, including patients with NAFLD, was completed by Tzefos and Olin. ${ }^{23}$ There were 12 trials reviewed and the authors concluded that statins were safe for the management of dyslipidemia in patients with NAFLD, including NASH.

In a retrospective study conducted by Maroni et $\mathrm{al}^{25}$ patients with a clinical diagnosis of NAFLD and dyslipidemia were evaluated for statin effects on the lipid profile and changes in liver enzyme concentrations. After $5.4 \pm 5.4$ months of therapy, total cholesterol, low-density lipoprotein cholesterol (LDL-C), and triglycerides significantly improved compared with baseline $(P<.0001$ for all parameters $)$. There were no significant changes in aspartate aminotransferase (AST), alanine aminotransferase (ALT), and $\gamma$ glutamyl transferase (GGT) concentrations $(P=.06, \quad P=.10, \quad$ and $P=.30$, respectively). Adverse events related to statins were not reported. This retrospective analysis demonstrated that statins still have a positive impact on the lipid panel without adversely affecting liver enzymes in patients with NAFLD.

In another study by Rana et al, ${ }^{26}$ statins were compared with insulin sensitizers in patients with NAFLD. No baseline characteristics were reported. No difference was noted for changes in weight between the groups at 24 weeks. At the end of 24 weeks, there was a significant difference between the groups for ALT, but not for AST. Cholesterol parameters were significantly reduced compared with baseline values in all groups. Statins had a significant reduction in ultrasonography (USG) scores at 24 weeks. The USG score was used to grade hepatic steatosis, with a score of 2 or more representing fatty liver. This study is unique in that it used active comparators to assess efficacy.

Similarly, in our study patients were evaluated at 3 months and 6 months with liver enzymes. Patients showed significant improvement in ALT levels. USG was repeated at 6 months and we found that patients on statins had significant improvement. Those on grade III moved to grade II and those on grade II moved to grade I. However, majority who took statins tolerated them well and very few experienced adverse effects. In fact, at currently recommended doses, an elevation of liver enzymes $>3$ times ULN, occurs in $<1 \%$ of treated patients ${ }^{[13],}{ }^{[14]}$. Moreover, it has also been highlighted that monitoring for hepatotoxicity is ineffective in predicting serious liver toxicity. Recently, a more aggressive statin therapy has been suggested as per ACC guidelines based on the results of randomized controlled trials (RCTs) showing a dramatically decrease of cardiovascular events in secondary prevention. ${ }^{27,28}$

In our study only $7.4 \%$ of patients taking statins experienced a rise in serum ALT $\geq 40 \mathrm{U} / \mathrm{L}$, and in each case the rise was transient, returning near baseline or below without discontinuation of statin treatment ${ }^{[43]}$. Similar findings were obtained in a small prospective study on the treatment of 23 hyperlipidaemic patients with biochemical and ecographic evidence of NAFLD, where rosuvastatin $10 \mathrm{mg} /$ day for 8 months normalized liver function tests in all patients (mean ALT from 90.9 to $29.8 \mathrm{U} / \mathrm{L}, p<0.001$ and mean AST from 91 to $30.5 \mathrm{U} / \mathrm{L}, p<0.001 .^{32}$

Similarly, in a study performed in 45 patients with NAFLD, type 2 diabetes and elevated LDL-C, a significant reduction was observed after 6 months of statin therapy. (mean ALT 66.58 vs. $29.46 \mathrm{U} / \mathrm{L}$, 
$p<0.0001$ and mean AST 59.61 vs. $24.00 \mathrm{U} / \mathrm{L}$, $p<0.0001){ }^{33}$

In a recent Cochrane meta-analysis on the effect of statins for NAFLD and NASH based on two trials with high risk of bias and a small numbers of participants, statins were found to improve serum aminotransferase levels as well as ultrasound findings ${ }^{29}$.

The effect of multifactorial treatment including lifestyle and drug treatment for risk factors in subjects with biochemical and ultrasonographic evidence of NAFLD was assessed in a randomized study performed in Greece. After 54 weeks of treatment, $67 \%$ in the atorvastatin, $42 \%$ in the fenofibrate and $70 \%$ in the combination treatment groups no longer had any surrogate evidence of NAFLD, as established by normalization of serum ALT plus normal liver echopattern. ${ }^{30}$ Similar findings were seen in our study.

Very recently, data from the population-based Rotterdam Study, including 2578 subjects who underwent liver ultrasonography, reported a lower prevalence of liver steatosis in patients treated with statins for more than 2 years. ${ }^{31}$

\section{Conclusion}

Statin therapy is safe in patients with NAFLD. The statin therapy did not increase the number of negative hepatic events. On the contrary, the liver enzyme levels normalized under statin treatment for patients with NAFLD, than for patients without statin treatment. Besides it also improved lipid profile and ultrsonographic findings thus reducing the number of cardiovascular events. Statin use for treatment of NAFLD is still controversial and off-label, but positive results have been shown for reductions in LFTs and USG reports. Outcomes for these trials vary, but all include some primary or secondary nonlipid outcomes focusing on hepatic clinical biochemical and/or ultrasound markers, which will help to add to the literature regarding the potential safety and efficacy of statins in the treatment of NAFLD. Continued research into the role of statins and hepatic outcomes in patients with NAFLD are further warranted.

Hence to conclude: Statins and non-alcoholic fatty liver disease, Important Facts-

1. Non-alcoholic fatty liver disease is the most common cause of incidental abnormal elevation of serum liver enzyme activities It is associated with dyslipidaemia and high risk for cardiovascular events.

2. Treatment with low to moderate intensity statins is safe. Preliminary studies have shown that statins may possibly improve hepatic histology in patients with underlying NAFLD

3. High intensity statin treatment is not recommended until necessary as it may induce liver toxicity.

4. Routine liver biochemistry monitoring in asymptomatic individuals is discouraged.

5. If ALT < 3xULN, continue therapy and recheck liver enzymes annually. If values rise $\geq 3 x U L N$, stop statin or reduce dose and re-check within 4-6 weeks.

6. The risk for serious liver injury from statins is quite rare and patients with NAFLD and hyperlipidaemia are not at increased risk for statin hepatotoxicity.

7. Previous recommendations advising against the use of statins in patients with dyslipidaemia and NAFLD were not evidence-based and should be reviewed with newer clinical trials.

\section{References}

1. Chalasani N, Younossi Z, Lavine JE, et al. The diagnosis and management of nonalcoholic fatty liver disease: practice guideline by the American Association for the Study of Liver Diseases, American College of Gastroenterology, and the American Gastroenterological Association. Hepatology. 2012;55:2005-2023. doi:10.1002/hep.25762. [PubMed] [CrossRef] [Google Scholar] 
2. Angulo P. Diagnosing steatohepatitis and predicting liver-related mortality in patients with NAFLD: two distinct concepts. Hepatology. 2011;53:17921794. doi:10.1002/hep.24403. [PubMed] [CrossRef] [Google Scholar]

3. Saadeh S, Younossi ZM, Remer EM, Gramlich T, Ong JP, Hurley M, et al. The utility of radiological imaging in nonalcoholic fatty liver disease. Gastroenterology 2002;123:745-50.

4. R. Loomba, A.J. SanyalThe global NAFLD epidemic Nature Reviews Gastroenterology \&Hepatology, 10 (2013), pp. 686-690Google Scholar

5. R. Pais, F. Charlotte, L. Fedchuk, et al.A systematic review of follow-up biopsies reveals disease progression in patients with non-alcoholic fatty liverJournal of Hepatology, 59 (2013), pp. 550-556 Google Scholar

6. G. Vernon, A. Baranova, Z.M. Younossi Systematic review: the epidemiology and natural history of non-alcoholic fatty liver disease and non-alcoholic steatohepatitis in adults Alimentary Pharmacology and Therapeutics, 34 (2011), pp. 274285Google Scholar

7. M.R. Souza, F. Diniz Mde, J.E. MedeirosFilho, et al.Metabolic syndrome and risk factors for non-alcoholic fatty liver disease Arquivos de Gastroenterologia, 49 (2012), pp. 89-96Google Scholar

8. S. Ballestri, A. Lonardo, S. Bonapace, et al.Risk of cardiovascular, cardiac and arrhythmic complications in patients with non-alcoholic fatty liver disease World Journal of Gastroenterology, 20 (2014), pp. 1724-1745Google Scholar

9. Younossi ZM, Koenig AB, Abdelatif D, Fazel Y, Henry L, Wymer M. Global epidemiology of nonalcoholic fatty liver disease-meta-analytic assessment of prevalence, incidence, and outcomes. Hepatology. doi:101002/hep.28431. [PubMed] [Google Scholar]

10. Lazo M, Clark JM. The epidemiology of nonalcoholic fatty liver disease: a global perspective. Semin Liver Dis. 2008;28:339-350. doi:10.1055/s-00281091978. [PubMed] [CrossRef] [Google Scholar]

11. Labrecque DR, Abbas Z, Anania F, et al. World Gastroenterology Organisation global guidelines: nonalcoholic fatty liver disease and nonalcoholic steatohepatitis. J Clin Gastroenterol. 2014;48:467-473. doi:10.1097/MCG.0000000000000116. [PubMed] [CrossRef] [Google Scholar]

12. P. Pignatelli, R. Carnevale, D. Pastori, et al.Immediate antioxidant and antiplatelet effect of atorvastatin via inhibition of Nox2 Circulation, 126 (2012), pp. 92103Google Scholar

13. F. Violi, C. Calvieri, D. Ferro, et al.Statins as antithrombotic drugs Circulation, 127 (2013), pp. 251-257Google Scholar

14. M. Del Ben, L. Polimeni, R. Carnevale, et al.NOX2-generated oxidative stress is associated with severity of ultrasound liver steatosis in patients with non-alcoholic fatty liver disease BMC Gastroenterology, 14 (2014), p. 81

15. V.G. Athyros, K. Tziomalos, T.D. Gossios, et al.Safety and efficacy of longterm statin treatment for cardiovascular events in patients with coronary heart disease and abnormal liver tests in the Greek Atorvastatin and Coronary Heart Disease Evaluation (GREACE) Study: a post-hoc analysis Lancet, 376 (2010), pp. 1916-1922

16. C.A. DujovneSide effects of statins: hepatitis versus "transaminitis"-myositis versus "CPK it is" American Journal of Cardiology, 89 (2002), pp. 14111413Google Scholar

17. Screening experience and baseline characteristics in the West of Scotland 
Coronary Prevention Study. The WOSCOPS Study Group. West of Scotland Coronary Prevention Study American Journal of Cardiology, 76 (1995), pp. 485-491Google Scholar

18. F.M. Sacks, M.A. Pfeffer, L. Moye, et al.Rationale and design of a secondary prevention trial of lowering normal plasma cholesterol levels after acute myocardial infarction: the Cholesterol and Recurrent Events trial (CARE) American Journal of Cardiology, 68 (1991), pp. 14361446Google Scholar

19. Design features and baseline characteristics of the LIPID (Long-Term Intervention with Pravastatin in Ischemic Disease) Study: a randomized trial in patients with previous acute myocardial infarction and/or unstable angina pectoris American Journal of Cardiology, 76 (1995), pp. 474-479

20. S. de Denus, S.A. Spinler, K. Miller, et al.Statins and liver toxicity: a metaanalysis Pharmacotherapy, 24 (2004), pp. 584-591

21. A.A. Alsheikh-Ali, P.V. Maddukuri, H. Han, et al. Effect of the magnitude of lipid lowering on risk of elevated liver enzymes, rhabdomyolysis, and cancer: insights from large randomized statin trials Journal of the American College of Cardiology, 50 (2007), pp. 409-418Google Scholar

22. K.M. Dale, C.M. White, N.N. Henyan, et al. Impact of statin dosing intensity on transaminase and creatine kinase American Journal of Medicine, 120 (2007), pp. 706-712

23. Tzefos M, Olin JL. 3-hydroxyl-3methylglutaryl coenzyme A reductase inhibitor use in chronic liver disease: a therapeutic controversy. J Clin Lipidol. 2011;5:450-459.

doi:10.1016/j.jacl.2011.06.013. [PubMed] [CrossRef] [Google Scholar]
24. de Keyser CE, Koehler EM, Schouten JN, et al. Statin therapy is associated with a reduced risk of non-alcoholic fatty liver in overweight individuals. Dig Liver Dis. 2014;46:720-725.

doi:10.1016/j.dld.2014.04.002. [PubMed] [CrossRef] [Google Scholar]

25. Maroni L, Guasti L, Castiglioni L, et al. Lipid targets during statin treatment in dyslipidemia patients affected by nonalcoholic fatty liver disease. Am J Med Sci. 2011;342:383-387. doi:10.1097/MAJ.0b013e318213e526. [PubMed] [CrossRef] [Google Scholar]

26. Rana H, Yadav SS, Reddy HD, Singhal S, Singh DK, Usman K. Comparative effect of insulin sensitizers and statin on metabolic profile and ultrasonographical score in non alcoholic fatty liver disease. $\mathbf{J}$ Clin Diagn Res. 2016;10:OC19-OC23.

27. J.C. LaRosa, S.M. Grundy, D.D. Waters, et al.Intensive lipid lowering with atorvastatin in patients with stable coronary disease New England Journal of Medicine, 352 (2005), pp. 14251435Google Scholar

28. M.A. Blazing, J.A. De Lemos, C.K. Dyke, et al. The A-to-Z Trial: methods and rationale for a single trial investigating combined use of low-molecular-weight heparin with the glycoprotein IIb/IIIa inhibitor tirofiban and defining the efficacy of early aggressive simvastatin therapy American Heart Journal, 142 (2001), pp. 211-217

29. L. Eslami, S. Merat, R. Malekzadeh, et al.Statins for non-alcoholic fatty liver disease and non-alcoholic steatohepatitis Cochrane Database of Systematic Reviews, 12 (2013), p. CD008623Google Scholar

30. V.G. Athyros, D.P. Mikhailidis, T.P. Didangelos, et al. Effect of multifactorial treatment on non-alcoholic fatty liver disease in metabolic syndrome: a 
randomised studyCurrent Medical Research and Opinion, 22 (2006), pp. 873883Google Scholar

31. C.E. de Keyser, E.M. Koehler, J.N. Schouten, et al. Statin therapy is associated with a reduced risk of nonalcoholic fatty liver in overweight individuals Digestive and Liver Disease, 46 (2014), pp. 720-725Google Scholar

32. S. Antonopoulos, S. Mikros, M. Mylonopoulou, et al.Rosuvastatin as a novel treatment of non-alcoholic fatty liver disease in hyperlipidemic patients Atherosclerosis, 184 (2006), pp. 233-234 Google Scholar

33. T. Abel, J. Feher, E. Dinya, et al.Safety and efficacy of combined ezetimibe/ simvastatin treatment and simvastatin monotherapy in patients with nonalcoholic fatty liver disease Medical Science Monitor, 15 (2009), pp. MS6MS11

34. Joseph AE, Saverymuttu SH, al-Sam S, Cook MG, Maxwell JD. Comparison of liver histology with ultrasonography in assessing diffuse parenchymal liver disease. Clin Radiol 1991;43:26-31.

35. Debongnie JC, Pauls C, Fievez M, Wibin E. Prospective evaluation of the diagnostic accuracy of liver ultrasonography. Gut 1981;22:130-5.

36. Saverymuttu SH, Joseph AE, Maxwell JD. Ultrasound scanning in the detection of hepatic fibrosis and steatosis. Br Med J (Clin Res Ed) 1986;292:13-5.

37. Webb M, Yeshua H, Zelber-Sagi S, Santo E, Brazowski E, Halpern $Z$, et al. Diagnostic value of a computerized hepatorenal index for sonographic quantification of liver steatosis. AJR Am J Roentgenol 2009;192:909-14.
38. Valls C, Iannacconne R, Alba E, Murakami T, Hori M, Passariello R, et al. Fat in the liver: Diagnosis and characterization. Eur Radiol 2006;16: 2292-30 